\title{
Association of Serum Soluble Receptor for Advanced Glycation End Products (sRAGE), S100A12, and VCAM-1 Levels with Further Post-Coronary Angiogram Endothelial Injury in Elderly and Younger Patients
}

\author{
Deeraj Mungun1, Iyan Zakaria1, Xiangming Wang1, Tiantian Tu1 ${ }^{1}$, Chuyan Feng1, Yan Guo ${ }^{1,2 *}$ \\ ${ }^{1}$ Department of Gerontology, The first affiliated hospital of Nanjing Medical University, Nanjing, China \\ ${ }^{2}$ Department of Cardiology, Jiangsu Shengze Hospital, Suzhou, China \\ *Correspondence to: Yan Guo, guoyan51@hotmail.com
}

How to cite this paper: Mungun, D., Zakaria, I., Wang, X.M., Tu, T.T., Feng, C.Y. and Guo, Y. (2018) Association of Serum Soluble Receptor for Advanced Glycation End Products (sRAGE), S100A12, and VCAM-1 Levels with Further Post-Coronary Angiogram Endothelial Injury in Elderly and Younger Patients. World Journal of Cardiovascular Diseases, 8, 48-58. https://doi.org/10.4236/wjcd.2018.81006

Received: August 9, 2017

Accepted: January 21, 2018

Published: January 24, 2018

Copyright $\odot 2018$ by authors and Scientific Research Publishing Inc. This work is licensed under the Creative Commons Attribution International License (CC BY 4.0).

http://creativecommons.org/licenses/by/4.0/ (c) (i) Open Access

\begin{abstract}
Objective: Coronary Angiography (CAG) refers to an aggressive process that is likely to lead to endothelial injury and possibly drive further interpretation of the cause for patients within current atherosclerotic illnesses. Therefore, this study aims to test certain correlations among concentration of sRAGE, VCAM-1, as well as S100A12 pre- and post-coronary angiography alongside likelihood of endothelial injury among various patients in different ages including the younger and the geriatric groups, thereby offering a full connection upon CAG impacts of patients having atherosclerosis. Method: In this study, the whole patients chosen had been grouped into two major groups on the basis of ages: the younger patient aged less than 65 years old (Group 1), and the elderly patient aged over 65 years old (Group 2). Soluble RAGE (sRAGE), VCAM-1 and S100A12 degrees in blood sampling gathered followed by measurements of CAG session for evaluating the inflammatory responses and likelihood of endothelial injury caused via CAG. All data was analyzed using SPSS version 21.0. Findings: Significant enhancement among concentration of serum VCAM-1 $(P=0.007)$ in younger patients and sRAGE $(P=0.019)$ in elderly patients had been observed, but that of serum S100A12 was still unaltered. Conclusions: Enhanced concentration of sRAGE and VCAM-1 post-CAG of patients having CAD are able to be related to follow-up endothelial injury, thus contributing to different pathway in exacerbating endothelial injury among younger and elderly patients.
\end{abstract}

\section{Keywords}

Coronary Angiography, Typical sRAGE, VCAM-1, S100A12 


\section{Introduction}

In China, Coronary Angiographies (CAG) has been commonly performed to investigate the severity of Coronary Artery Diseases (CAD). Although CAG has been a standardized medical operation, it is a typical invasive process. Soft plastic catheters applied in CAG are most likely to interfere normal endothelial injuries, or lead to follow-up atherosclerotic plaque information. Endothelial injury causes inflammatory reactions, which leads to inflammatory markers like sRAGE, S100A12, as well as VCAM-1.

RAGE (Receptor for Advanced Glycation End Products) belongs to the immunoglobulin super family and is expressed upon appearance of endothelial, lymphocytes as well as smooth muscle cells [1]. Full-length, $\mathrm{N}$-truncated as well as C-truncated soluble RAGEs are typical RAGEs [2] [3] [4]. Interaction between full-length RAGE and AGEs causes enhanced expression of adhesion molecules, covering soluble vascular cell adhesion molecule-1 (sVCAM-1) together with cytokine tumor necrosis factor-alpha (TNF- $\alpha$ ) [5] [6]. Conversely, activation of nuclear factor-kappa results in enhanced expression of pro-inflammatory genes [5] [7]; Circulating isoforms RAGE covering sRAGE is laminated from cell appearance via matrix metalloproteinases as well as endogenous secretory RAGE (esRAGE) [3] [4].

S100A12 refers to an inflammatory ligand of RAGE, which belongs to the S100 protein family. S100A12 adjusts inflammation and immune reaction [8]. Ligature of it within RAGE upon the endothelium, mononuclear phagocytes as well as lymphocytes drives cellular activation within emergence of primary pro-inflammatory mediators like: interleukin (IL)-1, tumor necrosis factor (TNF) and so on.

VCAM-1 is able to boost generation of leukocytes to endothelial cells, meanwhile accelerating migration of leukocytes along the endothelial appearance [8] [9]. Importantly, ascertaining concentration of VCAM-1 among arterial tissues, and elucidating correlation between arterial VCAM-1 expression and unstable endothelial homeostasis, can cause atherosclerosis' interpretation of the cause [10].

To supervise such inflammatory markers is crucial because they imply how CAG process might possibly intensify atherosclerosis' interpretation of the cause [11]. Therefore, purposes of this research are to confirm aforementioned risk by supervising the above biomarkers and contrasting every serum degree prior to and after CAG between elderly and younger patients. Consecutive clinical linkage between different concentrations of the discussed biomarker serums having likelihood of resultant endothelial injury among various patients may possibly build better foundations for follow-up medical appraisement.

\section{Patients and Methods}

In terms of sample size, the research covered 68 consecutive patients undergoing elective coronary angiography among which 47 were males and 21 were females 
aged between 46 to 81 years old. These samples were divided into two groups that Group 1 was made up of 34 younger patients aged less than 65 years old who underwent CAG procedure and having one stenosed coronary artery within over half of occlusion. In the meantime, Group 2 consisted of 34 elderly patients aged more than 65 years old who underwent CAG procedure and having one stenosed coronary artery within over half of occlusion. The target subjects sampled in the research comprise patients of Jiangsu Province Hospital, China. Besides, data was gathered from 07/2015 to 01/2016. Expressions of elective angiography covered patients having stable and unstable angina, having $\mathrm{CAD}$, and having nonspecific chest pain. Inclusion standards for the research subjects were on the basis of "ACC/AHA Directions for Coronary Angiography". Afterwards, patients having certain conditions were ruled out from samples. These conditions included: any malignance, kidney dysfunction, thyroid illness, severe coronary syndrome, autoimmune, as well as peripheral artery diseases. After acknowledgment of the research, comprehensive medical record for every patient has then been collected and combined. Besides, routine physical tests had then been implemented to build clinical diagnosis. Pilot written together with informed consent were gained from the whole patients; subsequently, the research got approval from Ethics Review Board of Jiangsu Province Hospital. The whole patients had been subjected by Cardiac Ultrasound Measurements. The systolic and diastolic blood pressure of every patient had been measured using the commonly applied mercury sphygmomanometer after at least ten minutes rest in sitting position. Body Mass Index (BMI) was estimated to be weight in kilograms classified via square of the height by meters. This research also paid attention to cardiovascular risk elements like smoking habits, high blood pressure as well as diabetes. In the 68 patients chosen, 33 patients were smoking people, 35 suffered from high blood pressure and 14 owned diabetes mellitus.

CAG had been conducted by means of radial method applying standardized angiographic measure. Before the angiography, the whole patients had been provided with Heparin 2000 IU IVP. Angiogram gathered had been conducted from 4 standardized projections for every coronary artery. Stenosis level decided in projection that revealed the determined in projection that showed the top serious shrinking. A total of three proficient interventional heart specialists had reviewed the whole cineangiograms and all of them were made unknown to specific study methods.

When it comes to the blood sample gathering process, two blood sample sets existed, including ones before, during and after operating of angiography session. First of all, blood gathering extracted $7 \mathrm{ml}$ of venous blood during the morning prior to operating and after a half day overnight fast and is majorly for underline blood profile analyses. Such samples had been drawn from antecubital vein and examined complying with standardized hospital process in Jiangsu Province Hospital. The other collection was blood elicitation prior to and after angiography session, gathered by a directing scabbard, and was majorly for measuring serum degree of sRAGE, VCAM-1, as well as S100A12. Every pa- 
tient's blood samples was centrifuged in $3000 \mathrm{rpm}$ for ten minutes at $4^{\circ} \mathrm{C}$ as well as deposited at $-59^{\circ} \mathrm{C}$ for separating serums from cells. Degrees of serum were decided via an enzyme-related immune sorbent assay kit approach.

The statistic results are presented in the form of mean \pm standardized deviation, which was analyzed using SPSS version 21.0. within t-test as well as below common distribution. Confrontation of underline features among groups is conducted by one-way ANOVAs. The two tests take advantage of two-tailed $P<$ 0.05 being a significant indicator of statistical discrepancies.

\section{Results}

Underline attributes of the whole patients are shown in Table 1. No significant discrepancies existed regarding in gender $(P=0.801)$, BMI $(P=0.182)$, systolic blood pressure $(P=0.254)$, diastolic blood pressure $(P=0.193)$, ejection fraction $(P=0.660)$, aspirin use $(P=0.109)$, beta-blocker use $(P=0.624), \mathrm{CCB}$ use $(P=$ $0.160)$, ACEI use $(P=0.325)$, statin use $(P=0.600)$, and nitrate use $(P=0.353)$ in that two groups of patients (see Table 1$)$. Such outcomes also denote that main cardiovascular risk elements, like smoking $(P=0.447)$, hypertension $(P=$ $0.282)$ as well as diabetes mellitus $(P=0.254)$, have no significant differences among two groups.

Table 1. Underline attributes among the three various patients groups.

\begin{tabular}{|c|c|c|c|}
\hline & $\begin{array}{c}\text { Group one } \\
\text { middle age population } \\
\text { age }<65 \text { years old } \\
(n=34)\end{array}$ & $\begin{array}{c}\text { Group two } \\
\text { elderly population } \\
\text { age }>65 \text { years old } \\
(n=34)\end{array}$ & $P$ value \\
\hline Age (years) & $55 \pm 4.4$ & $71 \pm 4.7$ & 0.000 \\
\hline Male/female & $24 / 10$ & $23 / 11$ & 0.801 \\
\hline BMI $\left(\mathrm{kg} / \mathrm{m}^{2}\right)$ & $25 \pm 3.1$ & $24 \pm 2.8$ & 0.182 \\
\hline Hypertension (\%) & $15(44)$ & $20(58)$ & 0.282 \\
\hline Diabetes mellitus (\%) & $9(26)$ & $5(14)$ & 0.254 \\
\hline Smoking (\%) & $15(44)$ & $18(52)$ & 0.447 \\
\hline $\mathrm{EF}(\%)$ & $60.3 \pm 8.8$ & $61.3 \pm 7.4$ & 0.660 \\
\hline Aspirin use (\%) & $24(71)$ & $17(50)$ & 0.109 \\
\hline Beta-blockers' use (\%) & $14(41)$ & $12(35)$ & 0.624 \\
\hline ACE inhibitors' use (\%) & $3(8)$ & $6(17)$ & 0.325 \\
\hline CCB use (\%) & $8(23)$ & $14(41)$ & 0.160 \\
\hline Statins use (\%) & $19(55)$ & $17(50)$ & 0.600 \\
\hline Nitrate use (\%) & $17(50)$ & $21(61)$ & 0.353 \\
\hline SBP (mmHg) & $123.4 \pm 17.6$ & $128.5 \pm 19.4$ & 0.254 \\
\hline $\mathrm{DBP}(\mathrm{mmHg})$ & $74.8 \pm 12.2$ & $71.8 \pm 11.9$ & 0.193 \\
\hline
\end{tabular}

Abbreviations: BMI, basal metabolism index; EF, ejection fraction; D-D, D-dimer; ACEI, angiotensin converter enzyme inhibitor; CCB, calcium channel blocker; SBP, systolic blood pressure; DBP, diastolic blood pressure. 


\section{Measurement of blood profile degrees among the whole patients.}

Preoperative blood profiles of the whole subjects are shown in Table 2. In the two groups, it can be seen that there is no significant differences among serum TC $(P=0.088)$, HDL-C $(P=0.219)$, LDL-C $(P=0.212)$, TG $(P=0.059), \mathrm{Hb}(P=$ $0.464)$, FPG $(P=0.091)$, as well as UA $(P=0.453)$ between two groups. Blood coagulation profiles (platelet $(P=0.178)$ and FIB $(P=0.977)$ ) in these two groups have no statistical difference, however D-D $(P=0.007)$ showed significant discrepancies between younger and elderly patients.

Outcomes are shown as the mean \pm standard deviation. For investigating the concentration of sRAGE, VCAM-1, as well as S100A12 in arterial blood prior to and after coronary angiography, immunohistochemistry was conducted. In group one, it is revealed in Table 3 that prior to coronary angiography, average

Table 2. Blood profile and lipid degrees in patients.

\begin{tabular}{|c|c|c|c|}
\hline & $\begin{array}{c}\text { Group one } \\
\text { middle age population } \\
\text { age }<65 \text { years old } \\
(n=34)\end{array}$ & $\begin{array}{c}\text { Group two } \\
\text { elderly population } \\
\text { age }>65 \text { years old } \\
(n=34)\end{array}$ & $P$ value \\
\hline $\mathrm{Hb}(\mathrm{g} / \mathrm{l})$ & $135.1 \pm 24.1$ & $143.2 \pm 57.8$ & 0.464 \\
\hline Platelet $\left(10^{9} / \mathrm{L}\right)$ & $215.8 \pm 79.3$ & $180.7 \pm 72.5$ & 0.178 \\
\hline $\mathrm{D}-\mathrm{D}(\mu \mathrm{g} / \mathrm{mL})$ & $0.42 \pm 0.49$ & $0.81 \pm 1.06$ & 0.007 \\
\hline Fibrinogen $(\mathrm{mmol} / \mathrm{l})$ & $2.7 \pm 0.69$ & $2.7 \pm 1.18$ & 0.977 \\
\hline $\mathrm{TC}(\mathrm{mmol} / \mathrm{l})$ & $4.2 \pm 0.91$ & $4.5 \pm 0.81$ & 0.088 \\
\hline HDL-C (mmol/l) & $1.4 \pm 1.7$ & $1.0 \pm 0.22$ & 0.219 \\
\hline LDL-C (mmol/l) & $2.7 \pm 0.7$ & $2.9 \pm 0.63$ & 0.212 \\
\hline TG (mmol/l) & $2.1 \pm 1.75$ & $1.5 \pm 0.66$ & 0.059 \\
\hline FGP (mmol/l) & $5.8 \pm 1.7$ & $5.2 \pm 1.1$ & 0.091 \\
\hline UA (mmol/l) & $351.1 \pm 106.5$ & $330 \pm 91.1$ & 0.453 \\
\hline
\end{tabular}

Abbreviations: $\mathrm{Hb}$, hemoglobin; D-D, D-dimer; TC, total cholesterol; HDL-C, high-density lipoprotein cholesterol; LDL-C, low-density lipoprotein cholesterol; TG, triglycerides; FGP, fasting plasma glucose; UA, uric acid.

Table 3. Concentration of S100A12, VCAM-1, sRAGE in arterial blood prior to and after CAG in younger patients.

\begin{tabular}{|c|c|c|c|}
\hline \multicolumn{3}{|c|}{$\begin{array}{c}\text { Group one (middle age population) } \\
\text { age }<65 \text { years old } \\
(n=34)\end{array}$} & \multirow[t]{2}{*}{$P$ value } \\
\hline Serum Protein & Average level before CAG & Average level after CAG & \\
\hline $\mathrm{S} 100 \mathrm{~S} 12(\mu \mathrm{g} / \mathrm{L})$ & $7.3 \pm 7.1$ & $7.1 \pm 6.9$ & 0.383 \\
\hline VCAM-1 ( $\mu \mathrm{g} / \mathrm{L})$ & $95.3 \pm 77.2$ & $114.3 \pm 92.5$ & 0.007 \\
\hline sRAGE (ng/L) & $95.3 \pm 78.5$ & $102.4 \pm 72.89$ & 0.093 \\
\hline
\end{tabular}

Abbreviations: VCAM-1, vascular cell adhesion molecule-1; sRAGE, soluble receptor of advanced glycation end products. 
concentration of sRAGE was $95.3 \pm 78.5 \mathrm{ng} / \mathrm{L}$, average concentration of VCAM-1 is $95.3 \pm 77.2 \mu \mathrm{g} / \mathrm{L}$, and average concentration of S100A12 is $7.3 \pm 7.1$ $\mu \mathrm{g} / \mathrm{L}$. After coronary angiography, average concentration of SRAGE is $102.4 \pm$ $72.89 \mathrm{ng} / \mathrm{L}$, average concentration of VCAM-1 is $114.3 \pm 92.5 \mu \mathrm{g} / \mathrm{L}$, and average concentration of S100A12 is $7.1 \pm 6.9 \mu \mathrm{g} / \mathrm{L}$. Significant enhancement in concentration of serum VCAM-1 $(P=0.007)$ in post CAG arterial blood can been seen. However, the findings of serum concentration S100A12 $(P=0.383)$ and sRAGE $(P=0.093)$ in post CAG arterial blood keeps comparatively uninterrupted.

In group two, it is revealed in Table 4 that prior to coronary angiography, average concentration of sRAGE is $83.8 \pm 53.1 \mathrm{ng} / \mathrm{L}, \mathrm{VCAM}-1$ is $77.4 \pm 59.4 \mu \mathrm{g} / \mathrm{L}$, and S100A12 is $6.0 \pm 5.0 \mu \mathrm{g} / \mathrm{L}$. After coronary angiography, average concentration of sRAGE is $94.1 \pm 45.5 \mathrm{ng} / \mathrm{L}, \mathrm{VCAM}-1$ is $77.9 \pm 53.1 \mu \mathrm{g} / \mathrm{L}$, and S100A12 is $6.3 \pm 5 \mu \mathrm{g} / \mathrm{L}$. Significant enhancement in concentration of serum sRAGE $(P=$ 0.019 ) in post CAG arterial blood can been seen. However, the findings of serum concentration S100A12 $(P=0.218)$ and VCAM-1 $(P=0.450)$ in post CAG arterial blood that keeps comparatively uninterrupted.

It can be seen that group two (elderly patients) possessed significant increased concentration of serum sRAGE among the post CAG arterial blood. Concentration of serum VCAM-1 among post CAG arterial blood of group 1 (younger patients) had been significantly promoted. Such outcomes indicated that either concentration of sRAGE or VCAM-1 in arterial blood had been significantly promoted in both younger patients and elderly patients with CAD who underwent CAG.

\section{Discussion}

Inflammation is also an essential component of atherosclerosis, as the process leads to the activation and recruitment of inflammatory cells, endothelial cell dysfunction and plaque formation [12]. During endothelial injury context, it is going to further induce pro-inflammatory reaction, linked with inflammatory biomarkers output.

Table 4. Concentration of of S100A12, VCAM-1, sRAGE in the arterial blood prior to and after CAG in elderly patients.

\begin{tabular}{cccc}
\hline & $\begin{array}{c}\text { Group two (elderly population) } \\
\text { age }>65 \text { years old } \\
(\boldsymbol{n}=34)\end{array}$ & & \\
\cline { 1 - 2 } Serum Protein & $\begin{array}{c}\text { Average level } \\
\text { before CAG }\end{array}$ & $\begin{array}{c}\text { Average level } \\
\text { after CAG }\end{array}$ & \\
\hline S100S12 $(\mu \mathrm{g} / \mathrm{L})$ & $6.0 \pm 5.0$ & $6.3 \pm 5$ & 0.218 \\
VCAM-1 $(\mu \mathrm{g} / \mathrm{L})$ & $77.4 \pm 59.4$ & $77.9 \pm 53.1$ & 0.450 \\
sRAGE $(\mathrm{ng} / \mathrm{L})$ & $83.8 \pm 53.1$ & $94.1 \pm 45.5$ & 0.019 \\
\hline
\end{tabular}

Abbreviations: VCAM-1, vascular cell adhesion molecule-1; sRAGE, soluble receptor of advanced glycation end products. 
VCAM-1 is an immunoglobulin-like adhesion molecule expressed on activated endothelial cells [13].

According to Weinkau et al., VCAM-1 is a crucial marker of vascular endothelial injury and the degrees are enhanced via indicating VCAM-1 expression among endothelial cells [14]. Several latest researches also show that large elevation among concentration of VCAM-1 is able to be motivated by the inflammatory cytokines [15] [16].

Enhanced expression of the VCAM-1 among vascular endothelial cells after CAG disseminates adherence as well as integration of leukocytes to vascular wall [17]. VCAM-1 motivates migration of appendiculate leukocytes alongside vascular endothelial appearance, and motivate diffusion of glossy muscle cells; as such, it can be supposed that VCAM-1 is able to be comprised in further pathogenesis of atherosclerosis because of endothelial injury [16] [18]. Saidi et al. [19] once claimed that among patients with atherosclerosis, harms of endothelial cells own correlation with elevation of VCAM-1 degree.

Regarding our findings, there is significant and independent correlation of enhanced concentration of VCAM-1, had been significantly higher after CAG in younger patients (group 1). However, the concentration of VCAM-1 in elderly patients (group 2) was slightly elevated after CAG, but not significant.

The outcomes of us are consistent with that of Saidi et al. The overall enhancement in concentration of VCAM-1 is linked with aggressive attribute of CAG process; and the large enhancement in concentration of VCAM-1 in group 1 sheds light on that with the increasing severity of atherosclerosis, the elevation in concentration of VCAM-1 is higher. As a result of this, CAG can possibly incite further atherosclerosis' pathogenesis among patients having more serious atherosclerotic plague.

While previous researches indicated that sRAGE can reflect increased activity among RAGE system as effects of ligand motivation mediate sRAGE up regulation [20] and sRAGE has been concealed in parallel with RAGE [21] [22]. The sRAGE is a RAGE's isoform that is short of the intracellular field, hence intracellular signaling [23]. On the contrary, sRAGE spreads in blood and is thought to indicate RAGE and inflammatory practice [24].

In addition, the sRAGE can be generated by two distinctive methods, endogenous secreted being a splice variant, esRAGE [22] or being a laminated variant [23] [24] [25]. A least expression of RAGE below regular conditions exists while it highly enhances in cellular pressure [26]. As discussed in the above part that sRAGE indicate RAGE practice.

Regarding this research, a significant and independent correlation of enhanced concentration of sRAGE, had been significantly higher after CAG in elderly (group 2). From a flip side, the concentration of sRAGE in younger (group 1) was slightly elevated after CAG, but not significant.

It can be found that significant enhancement of sRAGE after CAG in the group two revealed higher RAGE practice. Therefore, indicating higher inflam- 
matory practice that is linked to process of CAG. Additionally, VCAM-1 and RAGE are involved in the same immunoglobulin super family [5] [27], endothelial injury because of CAG process is able to motivate inflammatory response. Integration of inflammatory response and AGEs-RAGE communication induces VCAM-1 expression among endothelial cells [15] [16]. In elderly, CAG procedures may promote endothelial injury through significant enhancement of inflammatory process (RAGE practice) and secondary VCAM-1 expression among endothelial cells.

There are significant differences between the elderly and the younger in post CAG serum inflammatory biomarkers output, which reflects the degree of causes of vascular endothelial injury varies substantially across age period.

The observed enhancement in sRAGE and VCAM-1 has been assumed to be independent of any underline attributes, blood coagulation profile, and cardiovascular risk elements since no significant discrepancy exist between two groups (see Table 1 and Table 2$)$. Only D-D $(P=0.007)$ showed significant discrepancies between younger and elderly patients. It can be explained that elderly has higher risk of thrombus formation.

The vascular endothelium homeostasis plays an important role. Any structural or functional abnormality in the vascular wall (involving the extracellular matrix, vascular smooth muscle or endothelium) that occurs during aging may contribute to the increased risk of thrombosis in the elderly, particularly at hero thrombosis [28]. In addition, some researches show that sRAGE own higher affinity for heparin binding [19] [29] [30]. Because the whole patients are managed with heparin being part of CAG process, likelihood exists that concentration of sRAGE in post CAG-blood samples keeps lower than reality. In this case, it is foreseeable that large enhancement during concentration of sRAGE in elderly with additional risk of thrombus formation may require additional clinical management, such as administration of heparin post CAG procedure.

CAG process was aggressive diagnostic approach which share different mechanism of endothelial injury in elderly and younger patients. Especially in elderly, because of activation of inflammatory response and AGEs-RAGE axis induces VCAM-1 expression among endothelial cells. Such context will result in elderly has more pathways leading to endothelial injury. Combine with higher risk of thrombus formation, It can be hypothesized that group two may expose to higher risk of exacerbation in atherosclerosis plaque formation after CAG procedure.

No large shift exists during concentration of S100A12 prior to and after CAG. This may be caused via seizing of pro-inflammatory receptor as well as s100A12 [9]. Hence, indicating lower concentration of S100A12. An additional plausible interpretation would be because of the sample subjects' health profile. One research also revealed that a serum S100A12 focus keeps higher in patients having ACS than patients having steady CAD [31]. As subjects in the research are majorly patients having $\mathrm{CAD}$, degree of S100A12 cannot react as sensitive. 


\section{Conclusion}

A large and independent correlation of enhanced concentrations of sRAGE and VCAM-1, enhance the causation between endothelial injury and CAG process, particularly in patients with atherosclerotic illness. Therefore, CAG revealed both elderly and younger patients having CAD to larger risk of atherosclerosis that further exacerbates current status, although it reveals that both groups do not share the same pathogenesis pathway. Elderly has higher risk of greater exacerbation compared to the younger patients. As such, precautionary methods to guard against aforementioned risk should be conducted before, meanwhile and after CAG process, like additional use of heparin and others measures. Other non-aggressive diagnostic instruments are preferred by patients having CAD, like Cardiac ECT and dual source CT. However, future related clinical researches ought to be carried out for assessing the whole benefits of using non-aggressive diagnostic instruments rather than CAG. The research is practical in helping people aged over 65 years old to prevent themselves from vascular endothelial injury.

\section{Limitations}

Despite the achievements in present study, there are some limitations that must be highlighted. This research has employed a cross-sectional approach using a small sample size, thus affecting the external validity of research. Therefore, it is suggested for future researches to conduct multi-center prospective longitudinal researches upon bigger population to reconfirm the observation. Furthermore, this research has used the shifts in concentration sRAGE to be a direct indicator of RAGE practices. Further researches can measure expression of RAGE in a direct way. In addition, it may be crucial when these results were measured via expression of VCAM-1 upon arterial tissues rather than concentration of VCAM-1 among soluble pattern.

\section{References}

[1] Arsov, S., Graaff, R., van Oeveren, W., et al. (2014) Advanced Glycation End-Products and Skin Autofluorescence in End-Stage Renal Disease: A Review. Clinical Chemistry and Laboratory Medicine, 52, 11-20. https://doi.org/10.1515/cclm-2012-0832

[2] Bower, J.K., Pankow, J.S., Lazo, M., et al. (2014) Three-Year Variability in Plasma Concentrations of the Soluble Receptor for Advanced Glycation End Products (sRAGE). Clinical Biochemistry, 47, 132-134. https://doi.org/10.1016/j.clinbiochem.2013.11.005

[3] Foell, D., Wittkowski, H., Kessel, C., et al. (2013) Proinflammatory S100A12 Can Activate Human Monocytes via Toll-Like Receptor 4. American Journal of Respiratory and Critical Care Medicine, 187, 1324-1334. https://doi.org/10.1164/rccm.201209-1602OC

[4] López-Díez, R., Rastrojo, A., Villate, O., et al. (2013) Complex Tissue-Specific Patterns and Distribution of Multiple RAGE Splice Variants in Different Mammals. Genome Biology and Evolution, 512, 2420-2435. https://doi.org/10.1093/gbe/evt188 
[5] Mahajan, N. and Dhawan, V. (2013) Receptor for Advanced Glycation End Products (RAGE) in Vascular and Inflammatory Diseases. International Journal of Cardiology, 168, 1788-1794. https://doi.org/10.1016/j.ijcard.2013.05.013

[6] Saleh, A., Smith, D.R., Tessler, L., et al. (2013) Receptor for Advanced Glycation End-Products (RAGE) Activates Divergent Signaling Pathways to Augment Neurite Outgrowth of Adult Sensory Neurons. Experimental Neurology, 249, 149-159. https://doi.org/10.1016/j.expneurol.2013.08.018

[7] Kierdorf, K. and Fritz, G. (2013) RAGE Regulation and Signaling in Inflammation and Beyond. Journal of Leukocyte Biology, 94, 55-68.

https://doi.org/10.1189/jlb.1012519

[8] Adam, O., Marion, A. and Hofmann, B. (2015) S100A12 and the S100/Calgranulins Emerging Biomarkers for Atherosclerosis and Possibly Therapeutic Targets. Arteriosclerosis Thrombosis and Vascular Biology, 12, 2496-2506.

[9] Zhang, C. and Alirio, J.M. (2007) Role of Cell Adhesion Molecules and Immune-Cell Migration in the Initiation, Onset and Development of Atherosclerosis. Cell Adhesion \& Migration, 1, 171-175. https://doi.org/10.4161/cam.1.4.5321

[10] Mu, W., Chen, M.Y., Gong, Z.S., et al. (2015) Expression of Vascular Cell Adhesion Molecule-1 in the Aortic Tissues of Atherosclerotic Patients and the Associated Clinical Implications. Experimental and Therapeutic Medicine, 10, 423-428. https://doi.org/10.3892/etm.2015.2540

[11] Saadeddin, S.M., Habbab, M.A. and Ferns, G.A. (2002) Markers of Inflammation and Coronary Artery Disease. Medical Science Monitor, 8, RA5-RA12.

[12] Ross, R. (1999) Atherosclerosis-An Inflammatory Disease. The New England Journal of Medicine, 340, 115-126. https://doi.org/10.1056/NEJM199901143400207

[13] Osborn, L., et al. (1989) Direct Expression Cloning of Vascular Cell Adhesion Molecule 1, a Cytokine-Induced Endothelial Protein That Binds to Lymphocytes. Cell, 59, 1203-1211. https://doi.org/10.1016/0092-8674(89)90775-7

[14] Weinkauf, C.C., Berman, S.S., Goshima, K., et al. (2016) Vascular Cell Adhesion Molecule 1 Is a Promising Target to Identify High-Risk Carotid Plaques using Contrast-Enhanced Duplex Ultrasound. Journal of Vascular Surgery, 642, 549. https://doi.org/10.1016/j.jvs.2016.05.032

[15] Wrigley, B.J., Shantsila, E., Tapp, L.D., et al. (2011) Increased Expression of Cell Adhesion Molecule Receptors on Monocyte Subsets in Ischaemic Heart Failure. Thrombosis and Haemostasis, 1101, 92-100.

[16] Dörr, O., Liebetrau, C., Möllmann, H., et al. (2014) Soluble fms-Like Tyrosine Kinase-1 and Endothelial Adhesion Molecules (Intercellular Cell Adhesion Molecule-1 and Vascular Cell Adhesion Molecule-1) as Predictive Markers for Blood Pressure Reduction after Renal Sympathetic Denervation. Hypertension, 635, 984-990. https://doi.org/10.1161/HYPERTENSIONAHA.113.02266

[17] Schlesinger, M. and Bendas, G. (2015) Vascular Cell Adhesion Molecule-1 (VCAM-1) - An Increasing Insight into Its Role in Tumorigenicity and Metastasis. International Journal of Cancer, 13611, 2504-2514. https://doi.org/10.1002/ijc.28927

[18] Seropian, I.M., Toldo, S., Van Tassell, B.W., et al. (2014) Anti-Inflammatory Strategies for Ventricular Remodeling Following ST-Segment Elevation Acute Myocardial Infarction. Journal of the American College of Cardiology, 6316, 1593-1603. https://doi.org/10.1016/j.jacc.2014.01.014

[19] Saidi, H., Vakilian, M., Noori, G.H., et al. (2013) Alterations in Circulating Adhesion Molecules in Acute Myocardial Infarction before and after Thrombolysis with 
Streptokinase. Journal of Cardiovascular and Thoracic Research, 54, 139-141.

[20] Kang, J.H., Hwang, S.M. and Chung, I.Y. (2015) S100A8, S100A9 and S100A12 Activate Airway Epithelial Cells to Produce MUC5AC via Extracellular Signal-Regulated Kinase and Nuclear Factor- $\kappa$ B Pathways. Immunology, 1441, 79-90. https://doi.org/10.1111/imm.12352

[21] Bartels, S., Lori, M., Mbengue, M., et al. (2013) The Family of Peps and Their Precursors in Arabidopsis: Differential Expression and Localization but Similar Induction of Pattern-Triggered Immune Responses. Journal of Experimental Botany, 6417, 5309-5321. https://doi.org/10.1093/jxb/ert330

[22] Sterenczak, K.A., Nolte, I. and Escobar, H.M. (2013) RAGE Splicing Variants in Mammals. In: Heizmann, C.W., Ed., Calcium-Binding Proteins and RAGE: From Structural Basics to Clinical Applications, Humana Press, New York, 265-276.

[23] Piras, S., Furfaro, A.L., Domenicotti, C., et al. (2016) RAGE Expression and ROS Generation in Neurons: Differentiation versus Damage. Oxidative Medicine and Cellular Longevity, 2016, Article ID: 9348651. https://doi.org/10.1155/2016/9348651

[24] Schnitzer, J.E. and Oh, P. (2014) Tissue-Specific Imaging and Therapeutic Agents Targeting Proteins Expressed on Lung Endothelial Cell Surface. U.S. Patent 235, 8-26.

[25] Amin, A.R. and Islam, A.B. (2014) Genomic Analysis and Differential Expression of HMG and S100A Family in Human Arthritis: Upregulated Expression of Chemokines, IL-8 and Nitric Oxide by HMGB1. DNA and Cell Biology, 338, 550-565. https://doi.org/10.1089/dna.2013.2198

[26] Robinson, A.B., Johnson, K.A.D., Bennion, B.G., et al. (2012) RAGE Signaling by Alveolar Macrophages Influences Tobacco Smoke-Induced Inflammation. American Journal of Physiology-Lung Cellular and Molecular Physiology, 302, L1192-L1199.

[27] Zheng, Y., Yang, W., Aldape, K., et al. (2013) Epidermal Growth Factor (EGF)-Enhanced Vascular Cell Adhesion Molecule-1 (VCAM-1) Expression Promotes Macrophage and Glioblastoma Cell Interaction and Tumor Cell Invasion. Journal of Biological Chemistry, 288, 31488-31495. https://doi.org/10.1074/jbc.M113.499020

[28] Emanuele, P., Paolo, B., Serena, M.P. and Ida, M. (2011) Risk Factors for Venous and Arterial Thrombosis. Blood Transfusion, 9, 120-138.

[29] Hanford, L.E., Enghild, J.J., Valnickova, Z., et al. (2004) Purification and Characterization of Mouse Soluble Receptor for Advanced Glycation End Products (sRAGE). The Journal of Biological Chemistry, 279, 50019-50024.

[30] Yan, X., Anzai, A., Katsumata, Y., et al. (2013) Temporal Dynamics of Cardiac Immune Cell Accumulation Following Acute Myocardial Infarction. Journal of Molecular and Cellular Cardiology, 62, 24-35. https://doi.org/10.1016/j.yjmcc.2013.04.023

[31] Liu, J., Ren, Y.G., Zhang, L.H., Tong, Y.W. and Kang, L. (2014) Serum S100A12 Concentrations Are Correlated with Angiographic Coronary Lesion Complexity in Patients with Coronary Artery Disease. Scandinavian Journal of Clinical and Laboratory Investigation, 74, 149-154. https://doi.org/10.3109/00365513.2013.864786 